\title{
ABSORÇÃO E DISTRIBUIÇÃO DE NUTRIENTES EM PLANTIOS COMERCIAIS DE BAMBU (Bambusa vulgaris) NO NORDESTE DO BRASIL ${ }^{1}$
}

\author{
Serliete de Carvalho Mendes², Silmar Gonzaga Molica ${ }^{3}$, Rinaldo Luiz Caraciolo Ferreira ${ }^{3}$ e Gérman \\ Hugo Gutierrez Céspedes ${ }^{4}$
}

RESUMO - No Engenho Mamoaba, em Pedras de Fogo, PB, compararam-se a absorção e utilização de nutrientes em três povoamentos comerciais de Bambusa vulgaris, com diferentes declividades e densidades de plantas, sob a rotação anual de corte raso em solo arenoso. Na época de corte, em três parcelas de 15 x 15 m por povoamento, analisou-se o solo em três profundidades, e em três touceiras pesaram-se colmos novos e velhos, galhos, folhas e rizomas, além da manta orgânica, dos quais foram pesadas subamostras, antes e depois da secagem, para determinação da biomassa seca e do conteúdo de N, P, K, Ca e Mg. Na análise do conteúdo e da razão biomassa/conteúdo de cada nutriente, assumiu-se o delineamento inteiramente casualizado, e compararam-se as médias pelo teste de Tukey a 5\% de probabilidade. A declividade elevou a eficiência de absorção de P, K e Ca e a maior densidade de plantas, a eficiência de absorção de K. A eficiência de utilização de nutrientes na biomassa total e comercial foi similar nos três povoamentos. Nas folhas, o conteúdo de nutrientes não oscilou entre povoamentos, mas foi maior em N, e a sua eficiência variou para P. Na manta orgânica, os conteúdos de K, Ca e Mg foram maiores em declive, e com a utilização de $\mathrm{N}$ a eficiência foi menor. No rizoma, o conteúdo de nutrientes foi maior, sendo $\mathrm{N}$ o nutriente de uso menos eficiente. Como a ciclagem de nutrientes não compensa a exportação, a adubação é importante, principalmente com K, o mais exportado na biomassa.

Palavras-chave: Bambu, Bambusa vulgaris e Conteúdo de nutrientes.

\section{NUTRIENT ABSORPTION AND DISTRIBUTION IN COMMERCIAL STANDS OF BAMBOO (Bambusa vulgaris) IN THE NORTHEAST OF BRAZIL}

\begin{abstract}
In Pedras de Fogo-PB-Brazil, the absorption and the utilization of nutrients of three commercial stands of Bambusa vulgaris were compared in sandy soil with differential declivity and plant density, under annual total cut in sandy soil. In cut age, three $15 \times 15$ m plots were sampled by stand. In each plot, soil analysis in three levels of depths were made and three medium clumps were sampled and weighed old and new culms, twigs, leaves, rhizome and organic blanket. Sub samples of each biomass component were weighed, before and after drying, for dry biomass estimation and analysis of $N, P, K$, Ca and $M g$. The nutrient content and dry biomass / nutrient content ratio in each biomass component were analyzed by complete random design and the median was compared by Tukey test, with 5\% probability. The declivity raised the absorption efficiency of $P, K$ and $C a$, and higher clump density raised K absorption efficiency. The efficiency of nutrient utilization in total and commercial biomass was similar in all stands. In leaves, the nutrient content did not vary between stands, but was greatest for $N$, and the nutrient efficiency utilization varied for $P$. The nutrient content of felled leaves did not vary by stand, but was greater for $\mathrm{K}$, Ca and $\mathrm{Mg}$ in declivity and the nutrient efficiency of utilization was low for $N$. The rhizome had higher contents of nutrients and $N$ is the nutrient with lower utilization efficiency. Since the nutrient cycling did not compensate exportation, fertilization is necessary, mainly with $\mathrm{K}$, the most exported nutrient.
\end{abstract}

Keywords: Bamboo, Bambusa vulgaris and Mineral nutrients.

\footnotetext{
${ }^{1}$ Recebido em 19.11.2007 e aceito para publicação em 25.08.2010.

${ }^{2}$ INSTITUTO AGRONOMICO DE PERNAMBUCO, IPA, Brasil. E-mail: <serliete@hotmail.com>.

${ }^{3}$ Universidade Federal Rural de Pernambuco, UFRPE, Brasil. E-mail: <molicasilmar@hotmail.com>.

${ }^{4}$ Agro Industrial Mercantil Excelsior S/A, AGRIMEX, Brasil.
} 


\section{INTRODUÇÃO}

Na nutrição de plantas, há balanço em que parte dos nutrientes absorvidos é perdida com a exploração de biomassa (exportação do conteúdo na biomassa explorada), e parte dos nutrientes absorvidos é reposta ao solo por decomposição (reciclagem do conteúdo em resíduos). Assim, a manutenção da produtividade em solos de baixa fertilidade depende da reposição das quantidades de nutrientes exportados, especialmente sob baixa fertilidade, alta densidade de plantas e, ou, baixa idade de exploração (BARROS et al., 1981, 1982, 1990).

Esse balanço de nutrientes é afetado pela eficiência de absorção, definida pelo conteúdo de nutrientes na biomassa total, e da eficiência de utilização, definida pela razão biomassa/conteúdo de nutrientes nos componentes de biomassa explorados e reciclados. Essas eficiências são características genéticoadaptativas, sendo específicas para o nutriente em questão e se relacionam com fatores fisiológicos, como o genótipo e a idade das plantas, e com fatores ambientais, como tipo de solo e densidade de plantas (BARROS et al., 1986).

A avaliação nutricional do genótipo cultivado permite elevar a produtividade por: a) escolha de solos em que há melhor adaptação dos genótipos em razão de suas eficiências nutricionais; b) escolha de genótipos mais adaptados a solos nutricionalmente deficientes, por maior eficiência de absorção e utilização de nutrientes aplicados; c) utilização de manejo que reduza a exportação de nutrientes ao longo de ciclos de corte e, consequentemente, o uso de adubos (BARROS et al., 1990).

Em plantios de bambu, a densidade de plantas é alta, as rotações por corte raso são curtas e realizadas indefinidamente sem replantio, e a nutrição se distingue por ser caracterizada pela existência de um complexo de colmos sucessivamente conectados ao rizoma, levando à pressuposição de que, no planejamento da adubação, deve ser essencial a avaliação do status nutricional do sistema de cultivo.

Assim, estudaram-se, em plantios comerciais de Bambusa vulgaris no Nordeste do Brasil, a absorção, exportação e ciclagem de nutrientes, sob diferentes densidades de plantas, declividades do terreno e idades de colmos.

\section{MATERIAL E MÉTODOS}

\section{1. Área de Estudo}

O Engenho Mamoaba, pertencente à empresa Agrimex Agroindustrial Excelsior S. A., localiza-se na Bacia Hidrográfica do Rio Gramame, microrregião do litoral do Sul da Paraíba, no Município de Pedras de Fogo, PB, com área total de 2.284,12 ha, cultivados com bambu, em solo Podzólico Vermelho, de textura arenosa. A precipitação anual média é de 1.300 mm e a temperatura média anual, de $25 \mathrm{C}^{\circ}$.

\section{2. Definição dos Povoamentos sob Estudo}

Foram estudados, em maio de 2004, um ano após o último corte por talhadia, três tipos de povoamento mais comuns, conduzidos por rebrota após vários cortes anteriores por 22 anos, em solo arenoso, na mesma microbacia.

Os povoamentos I e II estavam no topo relativamente plano e tinham diferentes densidades de plantio, estabelecendo diferentes áreas úteis médias por touceira, que eram $3,75 \mathrm{~m}^{2}$ no povoamento I e $5,63 \mathrm{~m}^{2}$ no povoamento II. O povoamento III foi selecionado logo abaixo, em terreno inclinado, com declividade de 51,13\%, mas tinha área útil/touceira de $3,57 \mathrm{~m}^{2}$, similar à do povoamento $\mathrm{I}$.

Em cada povoamento foram selecionadas ao acaso três parcelas de 15 × 15 m.

\section{3. Caracterização Química do Solo}

A análise química do solo foi realizada para cada parcela, a partir de amostras compostas de cinco amostras simples coletadas separadamente com trado, em três níveis de profundidades (0-20; 20-40; e 40-60 cm), homogeneizadas, acondicionadas em sacos plásticos etiquetados, a serem enviadas ao laboratório (LAGRI).

Essa análise denota que o solo dos três povoamentos tem boa fertilidade, em razão da ciclagem da manta orgânica e da adubação anual feita conforme a análise do solo (Tabela 1). O fato de a fertilidade do solo em $\mathrm{P}$ ser alta em relação a K pode ser explicado pela maior absorção de $\mathrm{K}$, pois em várias pesquisas determinando os conteúdos médios de nutrientes na parte aérea do bambu, analisadas por Kleinhenz e Midmore (2001), encontrou-se a proporção 6 N : 1 P : 7 K. Nesse aspecto, o bambu seria similar a gramíneas, que, conforme Mello et al. (1987), têm alta capacidade de extração de K. Assim, a fertilidade do solo pode ter sido afetada pelo longo período de cultivo do bambu. 


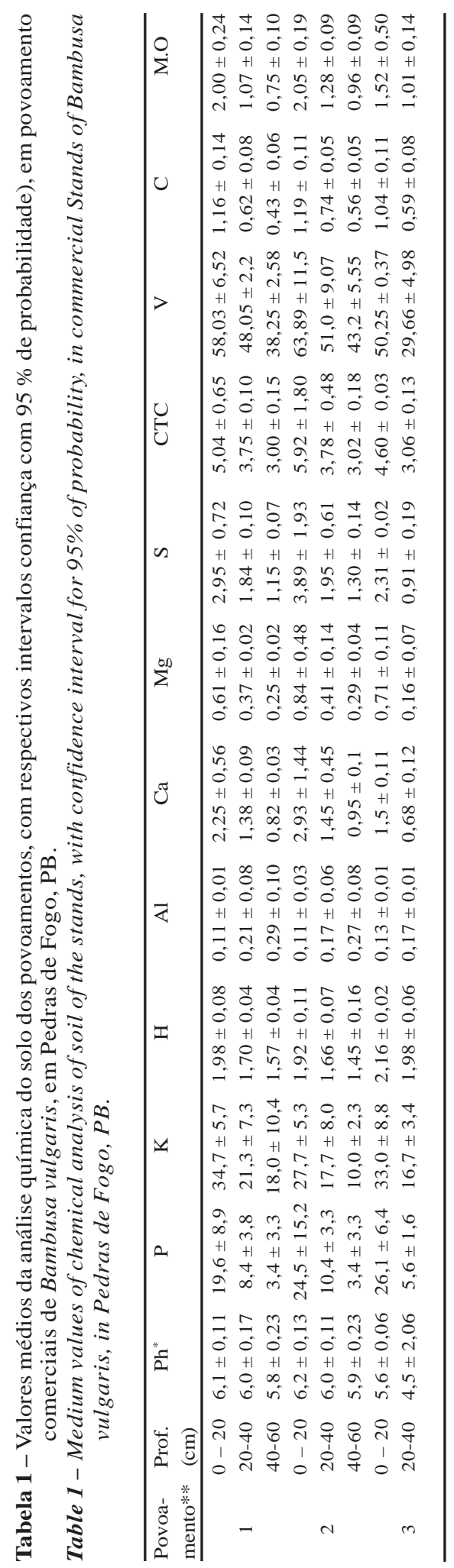

\section{4. Amostragem de Componentes de Biomassa}

Foram retiradas três touceiras medianas, com base no número, diâmetro e altura de colmos, de cada parcela, totalizando nove plantas por povoamento. As touceiras foram cortadas a $5 \mathrm{~cm}$ do solo, e os seus componentes de biomassa, folhas, galhos, colmos novos, colmos velhos e rizomas foram separados sobre uma lona plástica e pesados a campo em uma balança com capacidade para 150 kg, se necessário dentro de um saco plástico.

Após esse procedimento, para cálculo do teor de umidade de folhas, galhos, colmos novos, colmos velhos e rizoma foram retiradas, de cada um desses componentes, amostras com cerca de 100 g, que foram colocadas em sacos de papel Kraff, pesadas em balança de $700 \mathrm{~g}$ de capacidade e, no Laboratório de Melhoramento Genético da Usina Santa Teresa, secas em estufa de circulação forçada de ar, a $65^{\circ} \mathrm{C}$, até a estabilização do peso de matéria seca, e novamente pesadas visando à obtenção do peso da biomassa seca total de cada componente.

\section{5. Amostragem da Manta Orgânica por Povoamento}

A manta orgânica não decomposta sobre o solo de cada povoamento foi coletada como amostras compostas a partir de cinco subamostras ao acaso por parcela, obtidas sob um quadrado de madeira de 50 x $50 \mathrm{~cm}$, pesada a campo. Dessas amostras compostas pesadas a campo, após homogeneização, foram coletadas em sacos de papel amostras de cerca de 100 g, pesadas em balança mecânica, as quais foram levadas ao laboratório para secagem em estufa, a $65^{\circ} \mathrm{C}$, até peso constante. Após secagem, as amostras foram novamente pesadas, para cálculo da biomassa seca total.

\section{6. Análise de Nutrientes em Amostras Secas de Componentes das Touceiras}

As amostras secas das touceiras foram enviadas ao Laboratório LAGRI para análise química da concentração de nutrientes em cada componente de biomassa das touceiras.

Para o N, a concentração foi determinada em extrato sulfúrico de 0,2 g de material moído e, para P, K, Ca, $\mathrm{Mg}$ e $\mathrm{Na}$, em extrato da digestão nítrico-perclórica de $0,5 \mathrm{~g}$.

Nos extratos, determinaram-se $\mathrm{N}$ pelo método Kjeldahl, P, colorimetricamente, usando ácido ascórbico como redutor do complexo fosfomolíbdico (BRAGA 
e DEFELIPO, 1974), K por fotometria de emissão de chama e Ca e Mg por espectrofotometria de absorção atômica.

\section{7. Estimativa do Conteúdo de Nutrientes em Componentes da Touceira}

Os conteúdos de cada nutriente foram estimados por análise química das amostras secas de cada componente de biomassa das plantas amostrais. Essa estimativa foi obtida multiplicando-se o valor da biomassa seca de cada componente pela concentração de cada nutriente nesses componentes. O conteúdo de cada nutriente na biomassa aérea total foi considerado a eficiência de absorção. O balanço de nutrientes no sistema foi obtido relacionando o conteúdo de cada nutriente na biomassa explorada, que é considerada quantidade exportada de nutrientes, contra o conteúdo de nutrientes na biomassa devolvida ao solo (folhas), que é considerada quantidade reciclada de nutrientes e com a quantidade de cada nutriente contida no solo.

\section{8. Determinação do Coeficiente de Utilização Biológica - CUB}

O coeficiente de utilização de nutrientes foi calculado pelo coeficiente de utilização biológica (CUB), dividindose os valores de biomassa seca dos componentes vegetais pelo seu conteúdo de cada nutriente (BARROS et al., 1986), denotando o número de unidades de biomassa produzida por unidade de conteúdo de cada nutriente.

\section{Análise dos Resultados}

A distribuição do conteúdo de nutrientes e a razão biomassa/conteúdo de nutrientes, em cada um dos componentes das plantas, foram analisados adotando-se o delineamento inteiramente casualizado, com três tratamentos $=$ povoamentos $(\mathrm{i})$, três repetições $=$ parcelas (j) e três touceiras por parcela $(\mathrm{k})$, totalizando nove touceiras por tratamento e 27 touceiras totais. As médias foram comparadas pelo teste de Tukey a 5\% de probabilidade.

Os três povoamentos estavam na mesma microbacia, e os povoamentos I e III tinham similares densidades de plantas, mas o povoamento III estava situado em declive, e os povoamentos I e II tinham diferentes densidades de plantio, mas estavam localizados em terreno plano na mesma posição topográfica do topo da topossequência. Assim, realizou-se a comparação dos povoamentos aos pares (I com III e I com II), para discutir o efeito da declividade, da densidade de plantas e da idade dos colmos na absorção, exportação e reposição de nutrientes.

\section{RESULTADOS E DISCUSSÃO}

\section{1. Absorção de Nutrientes}

Para P, K e Ca, a eficiência de absorção foi maior no povoamento III, em declive (Tabela 2). Isso confirma Teixeira (1987), que justificou o maior conteúdo de nutrientes na biomassa total de plantas de Eucalyptus na parte baixa de topossequência pelo fato de esse local receber aporte de nutrientes por erosão. A eficiência de absorção para $\mathrm{N}$ e Mg foi similar em todos os povoamentos, provavelmente devido à variação entre plantas do mesmo povoamento.

A ausência de diferença na absorção dos nutrientes, com exceção de K, entre os povoamentos I e II, com diferentes densidades de plantas, pode ser explicada pela pouca idade da parte aérea das plantas, em rotação muito curta, que evita alta competição entre partes aéreas das plantas. Isso pode ter ocorrido porque, conforme Kleinhenz e Midmore (2001), o bambu tende a entrar em competição para produção da parte aérea após cerca de seis anos. Conforme Rezende et al. (1983) e França e Poggiani (1983), em Eucalyptus, menor espaçamento eleva o número de plantas por área, elevando também a quantidade total de nutrientes absorvidos, mas esse efeito se intensifica em idades de rotação mais altas.

Tabela 2 - Absorção de nutrientes, em kg/ha, com base no conteúdo na biomassa total (colmos novos e velhos, galhos, folhas e rizoma), em povoamentos comerciais de Bambusa vulgaris, em Pedras de Fogo, PB.

Table 2 - Absorption of Nutrients, in $\mathrm{Kg} / \mathrm{ha}$, with base in the Content in the Total Biomass (new and old stems, branches, leaves and rhizome), in Commercial Stands of Bambusa vulgaris, in Pedras de Fogo, PB.

\begin{tabular}{cccccc}
\hline Povoamentos & $\mathrm{N}$ & $\mathrm{P}$ & $\mathrm{K}$ & $\mathrm{Ca}$ & $\mathrm{Mg}$ \\
\hline I & 219,1 A & $25,5 \mathrm{~B}$ & $215,9 \mathrm{~A}$ & $58,8 \mathrm{AB}$ & $36,1 \mathrm{~A}$ \\
II & 145,1 A & $15,8 \mathrm{~B}$ & $77,3 \mathrm{~B}$ & $37,9 \mathrm{~B}$ & $15,5 \mathrm{~A}$ \\
III & $175,2 \mathrm{~A}$ & $37,3 \mathrm{~A}$ & $335,0 \mathrm{~A}$ & $74,8 \mathrm{~A}$ & $35,4 \mathrm{~A}$ \\
C. V. (\%) & 52,02 & 60,15 & 48,36 & 46,61 & 60,77 \\
\hline
\end{tabular}

Médias seguidas pela mesma letra não diferem significativamente entre si, pelo teste de Tukey $(\mathrm{P}>0,05)$.

Means followed by same letter do not differ significantly by Tukey test $(P>0.05)$. 
A maior absorção sob maior densidade de plantas no povoamento I, para $\mathrm{K}$, deve ter ocorrido porque esse nutriente apresenta o maior requerimento e maior conteúdo na biomassa total de bambus, em geral na proporção $7 \mathrm{~K}: 6 \mathrm{~N}$ : 1 P (KLEINKHENZ e MIDMORE, 2001).

\section{2. Exportação de Nutrientes}

Houve maior tendência à exportação de nutrientes na biomassa explorada (colmos e galhos) no povoamento III, na inclinação do terreno. Assim, a declividade pode ter elevado a exportação de nutrientes, em decorrência do carreio de nutrientes pela água das chuvas, que eleva a fertilidade e a produtividade da biomassa aérea explorada.

Não houve diferença na exportação de nutrientes entre os povoamentos I e II, com diferentes densidades de plantas. Isso indica que, na idade de rotação precoce adotada, o espaçamento não afeta a exportação de nutrientes por hectare (Tabela 3). A rotação curta, ao regredir as partes aéreas das plantas no estágio inicial de crescimento, pode ter eliminado a competição por espaço aéreo, nivelando o conteúdo de nutrientes na biomassa exportada. Isso também pode ter ocorrido por que menor número de plantas em maior espaçamento pode ser compensado por maior tamanho das touceiras (KLEINKHENZ e MIDMORE, 2001).

Entre os nutrientes exportados, o K é o mais representativo, por estar mais presente em partes exploradas (Tabela 3), confirmando a literatura mundial,

Tabela 3 - Exportação de nutrientes por povoamento, em $\mathrm{kg} / \mathrm{ha}$, com base no conteúdo de nutrientes na biomassa explorada (colmos e galhos), em povoamento comerciais de Bambusa vulgaris, em Pedras de Fogo, PB.

Table 3 - Exportation of Nutrients for stands, in $\mathrm{kg} /$ hectare, based on the Content of Nutrients in the Explored Biomass (stems and branches), in Commercial Stands of Bambusa vulgaris, in Pedras de Fogo, PB.

\begin{tabular}{cccccc}
\hline Povoamentos & $\mathrm{N}$ & $\mathrm{P}$ & $\mathrm{K}$ & $\mathrm{Ca}$ & $\mathrm{Mg}$ \\
\hline I & $32,5 \mathrm{~B}$ & $9,1 \mathrm{~A}$ & $80,2 \mathrm{~B}$ & $12,3 \mathrm{~B}$ & $9,1 \mathrm{AB}$ \\
II & $19,1 \mathrm{~B}$ & $6,0 \mathrm{~A}$ & $45,7 \mathrm{~B}$ & $6,3 \mathrm{~B}$ & $2,3 \mathrm{~B}$ \\
III & $52,9 \mathrm{~A}$ & $22,0 \mathrm{~A}$ & $220,9 \mathrm{~A}$ & $31,2 \mathrm{~A}$ & $16,8 \mathrm{~A}$ \\
C. V. $(\%)$ & 47,5 & 157,0 & 60,7 & 45,5 & 69,5 \\
\hline
\end{tabular}

Médias seguidas pela mesma letra não diferem significativamente entre si, pelo teste de Tukey $(\mathrm{P}>0,05)$.

Means followed by same letter do not differ significantly by Tukey test $(P>0.05)$. em que o maior conteúdo na biomassa aérea é de K (KLEINKHENZ e MIDMORE, 2001). A maior absorção pode ser a causa do baixo teor de $\mathrm{K}$ em relação aos outros nutrientes na análise do solo (Tabela 1).

Entre os componentes de biomassa exportados (colmos velhos e novos e galhos), colmos novos são importantes exportadores de nutrientes (Tabela 4). Assim, para reduzir a fase de crescimento lento centrado na produção de folhas, o corte raso, usado atualmente, deve ser comparado ao corte seletivo que mantém certo número de colmos novos com folhas. Houve semelhança entre colmos velhos e novos na tendência à exportação de nutrientes. Isso ocorreu, provavelmente, devido à baixa idade das plantas e à relativamente baixa quantidade de colmos novos nessa idade, pois, com o aumento da idade das plantas, a relação conteúdo de nutrientes/biomassa tende a diminuir (SHANMUGHAVEL e FRANCIS, 1996).

A eficiência de utilização de nutrientes para produção de cada componente de biomassa é maior quanto maior o número de unidades de biomassa produzida por unidade de conteúdo de nutrientes, denotando baixas concentrações. Assim, a eficiência de utilização de nutrientes é maior quanto maior a produção de biomassa e quanto menor a concentração ou o conteúdo de nutrientes nessa biomassa (BARROS et al., 1990).

Para produção de biomassa aérea explorada, não houve variação na eficiência de utilização de nutrientes entre povoamentos que são diferentes em declividade e em densidade de plantas (Tabela 5). Isso pode ser devido à baixa idade das plantas, em que o conteúdo de nutrientes, sendo processado na biomassa explorada, é maior do que em idades mais altas.

Assim, o povoamento mais produtivo não é necessariamente o mais eficiente na utilização de nutrientes, como ocorreu no local de maior declividade (povoamento III), em que, provavelmente, há maior aporte de nutrientes. Como nas plantas não houve diferença em produção em função da densidade de plantas por ha (povoamento I), a produtividade dependeu mais da eficiência de absorção do que da eficiência de utilização de nutrientes, em decorrência da oferta de nutrientes no solo e da baixa idade das plantas, em que há relativamente alto conteúdo de nutrientes sendo processado nos componentes de biomassa. Como a nutrição não limita o crescimento da parte aérea, o conteúdo de nutrientes pode acompanhar a produção de biomassa aérea, de modo que a eficiência de utilização varia pouco entre os povoamentos.

Revista Árvore, Viçosa-MG, v.34, n.6, p.991-999, 2010 
Tabela 4 - Conteúdo médio (kg/ha) de nutrientes em colmos novos, colmos velhos e galhos, em povoamentos comerciais de Bambusa vulgaris, em de Pedras de Fogo, PB.

Table 4-Medium content ( $\mathrm{kg} / \mathrm{ha}$ ) of nutrients in new stems, old stems and branches, of commercial stands of Bambusa vulgaris, in Pedra de Fogo, PB.

\begin{tabular}{|c|c|c|c|c|c|c|c|c|c|c|c|c|c|c|c|}
\hline \multirow[t]{2}{*}{ Povoamentos } & \multicolumn{5}{|c|}{ Colmos Novos } & \multicolumn{5}{|c|}{ Colmos Velhos } & \multicolumn{5}{|c|}{ Galhos } \\
\hline & $\mathrm{N}$ & $\mathrm{P}$ & $\mathrm{K}$ & $\mathrm{Ca}$ & $\mathrm{Mg}$ & $\mathrm{N}$ & $\mathrm{P}$ & $\mathrm{K}$ & $\mathrm{Ca}$ & $\mathrm{Mg}$ & $\mathrm{N}$ & $\mathrm{P}$ & $\mathrm{K}$ & $\mathrm{Ca}$ & $\mathrm{Mg}$ \\
\hline I & $10,6 \mathrm{~A}$ & $2,7 \mathrm{~B}$ & $31,0 \mathrm{~B}$ & $3,7 \mathrm{~B}$ & $2,8 \mathrm{AB}$ & $9,5 \mathrm{~B}$ & $3,2 \mathrm{~B}$ & $23,0 \mathrm{~B}$ & $4,6 \mathrm{~B}$ & $3,4 \mathrm{AB}$ & $12,3 \mathrm{AB}$ & $3,2 \mathrm{~B}$ & $26,2 \mathrm{~B}$ & $4,04 \mathrm{~B}$ & $2,9 \mathrm{AB}$ \\
\hline II & $7,1 \mathrm{~A}$ & $2,1 \mathrm{~B}$ & $20,4 \mathrm{~B}$ & $2,7 \mathrm{~B}$ & $0,9 \mathrm{~B}$ & $7,1 \mathrm{~B}$ & $1,8 \mathrm{~B}$ & $7,8 \mathrm{~B}$ & $1,9 \mathrm{~B}$ & $0,7 \mathrm{~B}$ & $4,9 \mathrm{~B}$ & $2,1 \mathrm{~B}$ & $17,5 \mathrm{~B}$ & $1,7 \mathrm{~B}$ & $0,7 \mathrm{~B}$ \\
\hline III & $7,1 \mathrm{~A}$ & $7,5 \mathrm{~A}$ & $89,0 \mathrm{~A}$ & $12,8 \mathrm{~A}$ & $6,9 \mathrm{~A}$ & $21,6 \mathrm{~A}$ & $7,1 \mathrm{~A}$ & $60,3 \mathrm{~A}$ & $9,2 \mathrm{~A}$ & $4,9 \mathrm{~A}$ & $24,2 \mathrm{~A}$ & $7,4 \mathrm{~A}$ & $71,5 \mathrm{~A}$ & $9,2 \mathrm{~A}$ & $5,0 \mathrm{~A}$ \\
\hline C. V. (\%) & 58,4 & 79,4 & 94,5 & 86,6 & 107,0 & 42,1 & 45,8 & 62,8 & 58,9 & 78,9 & 73,91 & 66,4 & 72,3 & 63,7 & 87,0 \\
\hline
\end{tabular}

Médias seguidas pelas mesmas letras não diferem significativamente entre si, pelo teste de Tukey $(\mathrm{P}>0,05)$.

Means followed by same letter do not differ significantly by Tukey test $(P>0.05)$.

Tabela 5 - Eficiência de utilização de nutrientes (kg biomassa seca/kg nutriente) na formação de colmos novos, colmos velhos e galhos dos povoamentos de Bambusa vulgaris, em Pedras de Fogo, PB.

Table 5 - Efficiency use of nutrients ( $\mathrm{kg}$ dry biomass $/ \mathrm{kg}$ nutrient) in the formation of new stems, old stems and branches of the stands of Bambusa vulgaris, in Pedras de Fogo, PB.

\begin{tabular}{|c|c|c|c|c|c|c|c|c|c|c|c|c|c|c|c|}
\hline \multirow[t]{2}{*}{ Povoamentos } & \multicolumn{5}{|c|}{ Colmos novos } & \multicolumn{5}{|c|}{ Colmos velhos } & \multicolumn{5}{|c|}{ Galhos } \\
\hline & $\mathrm{N}$ & $\mathrm{P}$ & $\mathrm{K}$ & $\mathrm{Ca}$ & $\mathrm{Mg}$ & $\mathrm{N}$ & $\mathrm{P}$ & $\mathrm{K}$ & $\mathrm{Ca}$ & $\mathrm{Mg}$ & $\mathrm{N}$ & $\mathrm{P}$ & K & $\mathrm{Ca}$ & Mg \\
\hline I & $230,0 \mathrm{~B}$ & $917,6 \mathrm{~A}$ & $83,9 \mathrm{~A}$ & 629,4 A & $1062,6 \mathrm{~B}$ & $281,3 \mathrm{~A}$ & $133,0 \mathrm{~B}$ & 116,7 B & 603,4 A & 961,5 B & 315,6 A & $1508 \mathrm{~A}$ & $181,2 \mathrm{~A}$ & 995,1 A & $2051 \mathrm{~A}$ \\
\hline II & $442,1 \mathrm{AB}$ & $871,6 \mathrm{~A}$ & $88,8 \mathrm{~A}$ & $670,4 \mathrm{~A}$ & $2827,2 \mathrm{~A}$ & 211,7 B & $822,9 \mathrm{~A}$ & $208,0 \mathrm{~A}$ & $758,2 \mathrm{~A}$ & $3078,5 \mathrm{~A}$ & $440,4 \mathrm{~A}$ & $4331 \mathrm{~A}$ & $539,1 \mathrm{~A}$ & $1156,9 \mathrm{~A}$ & $2843 \mathrm{~A}$ \\
\hline III & 925,9 A & $872,7 \mathrm{~A}$ & $72,6 \mathrm{~A}$ & 479,6 A & 1059,3 B & 255,6 BA & $778,2 \mathrm{~A}$ & $96,0 \mathrm{~B}$ & $627,2 \mathrm{~A}$ & 1283,7 B & $181,5 \mathrm{~A}$ & $569 \mathrm{~A}$ & $65,1 \mathrm{~A}$ & $483,1 \mathrm{~A}$ & 966 A \\
\hline C. V. (\%) & 98,21 & 46,36 & 28,44 & 29,81 & 57,50 & 19,76 & 48,43 & 24,92 & 23,17 & 57,56 & 126,66 & 275,36 & 291,44 & 153,14 & 159,87 \\
\hline
\end{tabular}

Médias seguidas pelas mesmas letras não diferem significativamente entre si, pelo teste de Tukey $(\mathrm{P}>0,05)$.

Means followed by same letter do not differ significantly by Tukey test $(P>0.05)$.

Isso também é explicado por resultados de Rezende et al. (1983) e França e Poggiani (1983), que trabalharam com eucalipto, em que a densidade de plantio, se não restringir a extração de nutrientes ou a produção de biomassa, pode não afetar a eficiência da utilização de nutriente, que tende a aumentar sob densidades que tendem a limitar a absorção sem restringir o crescimento das plantas.

A eficiência de utilização de nutrientes na produção de componentes de biomassa aérea explorada variou mais entre nutrientes, sendo a eficiência de utilização de K menor, por seu maior acúmulo nessa biomassa. O Ké um elemento que pode estar sendo temporariamente armazenado nos colmos para uso em momentos específicos, sendo o elemento com menor eficiência de uso pela planta e mais exportado do solo pela exploração de biomassa. Assim, para Kleinhenz e Midmore (2001) o K é elemento-chave na adubação do bambu, e, na formulação de fertilizantes deve ter participação proporcionalmente maior $(\mathrm{K}>\mathrm{N}>\mathrm{P}$ ). Nessa adubação, pode-se usar o cálculo indicado por Barros et al. (1988), baseado no conteúdo na biomassa explorada, expresso como conteúdo requerido no solo, descontando-se o conteúdo obtido por análise química do solo.

\section{3. Reposição de Nutrientes}

A reposição de nutrientes ao solo conforme o conteúdo na biomassa deixada no campo (folhas) não variou entre povoamentos. Isso pode ter ocorrido pela baixa idade das plantas, em que o processamento de nutrientes é alto, podendo haver consumo de luxo (Tabela 6).

Os conteúdos de K e N foram maiores em relação aos outros nutrientes, em razão do maior requerimento nas folhas. Embora a concentração e conteúdo de nutrientes em componentes de parte aérea variam muito com o genótipo, com a idade, com o ambiente e com a parte da planta, usando como referência a concentração em folhas, que é normalmente alta, no bambu, em diferentes espécies, idades e locais, há a tendência para a sequência $\mathrm{K}>\mathrm{N}>\mathrm{Ca}>\mathrm{Mg}>\mathrm{P}$ (KLEINHENZ e MIDMORE, 2001; SHANMUGHAVEL e FRANCIS, 2002; EMBAYE et al., 2005).

Devido à baixa biomassa e alto conteúdo de nutrientes nas folhas, altas concentrações resultam em valores relativamente mais baixos de eficiência de utilização, particularmente para K e N, que apresentaram maiores conteúdos nas folhas (Tabela 6). 
Tabela 6 - Conteúdo nédio e CUB de nutrientes nas folhas dos povoamentos comerciais de Bambusa vulgaris, em Pedras de Fogo, PB.

Table 6 - Medium content and CUB of nutrients in the leaves of the commercial stands of Bambusa vulgaris, in Pedras de Fogo, $P B$.

\begin{tabular}{|c|c|c|c|c|c|c|c|c|c|c|}
\hline \multirow[t]{2}{*}{ Povoamentos } & \multicolumn{5}{|c|}{ Conteúdo de nutrientes (kg/ha) } & \multicolumn{5}{|c|}{ CUB (kg de matéria seca/kg nutriente) } \\
\hline & $\mathrm{N}$ & $\mathrm{P}$ & $\mathrm{K}$ & $\mathrm{Ca}$ & $\mathrm{Mg}$ & $\mathrm{N}$ & $\mathrm{P}$ & $\mathrm{K}$ & $\mathrm{Ca}$ & $\mathrm{Mg}$ \\
\hline I & $68,1 \mathrm{~A}$ & $5,6 \mathrm{AB}$ & $53,3 \mathrm{~A}$ & $13,7 \mathrm{~A}$ & $7,9 \mathrm{~A}$ & $46,9 \mathrm{~A}$ & $669,7 \mathrm{~A}$ & $60,3 \mathrm{~A}$ & $235,5 \mathrm{~A}$ & $413,3 \mathrm{~A}$ \\
\hline II & $34,4 \mathrm{~A}$ & $2,1 \mathrm{~A}$ & $23,1 \mathrm{~A}$ & $7,7 \mathrm{~A}$ & $4,6 \mathrm{~A}$ & $42,1 \mathrm{~A}$ & $682,4 \mathrm{~A}$ & $61,2 \mathrm{~A}$ & 191,8 A & 318,9 A \\
\hline III & $55,2 \mathrm{~A}$ & 5,9 A & 56,6 A & $9,1 \mathrm{~A}$ & $9,5 \mathrm{~A}$ & $71,3 \mathrm{~A}$ & 508,3 В & 59,2 A & 1193,7 A & 403,6 A \\
\hline C. V. (\%) & 59,5 & 64,3 & 64,05 & 64,2 & 70,5 & 65,9 & 17,9 & 18,2 & 202,4 & 43,7 \\
\hline
\end{tabular}

Médias seguidas pela mesma letra não diferem significativamente entre si, pelo teste de Tukey $(\mathrm{P}>0,05)$.

Means followed by same letter do not differ significantly by Tukey test $(P>0.05)$.

A eficiência de utilização de nutrientes nas folhas variou entre povoamentos apenas para fósforo, sendo o povoamento III, com maior biomassa, menos eficiente (Tabela 6). Talvez isso ocorra pelo fato de o sistema radicular desse povoamento ter expandido mais, estando sob competição, mas com alta capacidade de absorção por contato, o que reduz a eficiência de utilização desse elemento. Segundo Mello et al. (1987), sistema radicular mais expandido facilita a absorção de $P$, pois a sua dinâmica de absorção ocorre mais pela eficiência de distribuição do sistema radicular e difusão em curta distância do que por fluxo de massa que acompanha a transpiração, como acontece com os outros nutrientes.

Pelo alto conteúdo de nutrientes nas folhas repostas ao solo, essa biomassa é essencial à manutenção da fertilidade, tendo o bambu alta eficiência de absorção de nutrientes e de acúmulo de biomassa e alta deposição de litter (CHISTANTY et al., 1996; ARUNACHALAM eARUNACHALAM, 2002; EMBAYE et al., 2005). Porém, como a quantidade reposta ao solo não superou as quantidades exportadas nos componentes de biomassa explorada, em particular em rotação curta desse caso, será necessária a reposição por meio de adubação em futuras rotações.

\section{4. Mineralização de Nutrientes}

O conteúdo na manta orgânica, em particular de $\mathrm{K}$, Ca e Mg, foi maior no povoamento III, denso e em terreno inclinado, apesar de não ter havido diferenças significativas para conteúdos em folhas. Assim, o fato de o conteúdo a ser devolvido ao solo ter diferido entre povoamentos pode ser devido a fatores externos às plantas, como menor atividade biológica na decomposição de resíduos orgânicos pela maior disponibilidade de nutrientes no solo, em decorrência do carreio pelas chuvas de partes mais altas (Tabela 7).

Comparando os povoamentos I e II, a deposição de nutrientes em folhas sobre o solo não variou em diferentes densidades de plantas. Assim, a densidade de plantas não afetou o conteúdo depositado no solo pelas folhas. Provavelmente pela baixa idade das plantas, a parte aérea tem conteúdos de nutrientes relativamente altos, com certo consumo de luxo. Assim, sob espaçamentos mais amplos as folhas teriam maior quantidade de nutrientes para depositar sobre o solo, compensando a menor quantidade de folhas.

A baixa quantidade de nutrientes na manta orgânica pode decorrer da rápida decomposição sob condições tropicais de umidade e temperatura, que depende do

Tabela 7 - Conteúdo médio e CUB de nutrientes na manta orgânica, em povoamentos comerciais de Bambusa vulgaris, em Pedras de Fogo, PB.

Table 7 - Medium content and CUB of nutrients in the organic blanket, in commercial stands of Bambusa vulgaris, in Pedras de Fogo, PB.

\begin{tabular}{|c|c|c|c|c|c|c|c|c|c|c|}
\hline \multirow[t]{2}{*}{ Povoamentos } & \multicolumn{5}{|c|}{ Conteúdo de nutrientes (kg/ha) } & \multicolumn{5}{|c|}{ CUB (kg de matéria seca/kg nutriente) } \\
\hline & $\mathrm{N}$ & $\mathrm{P}$ & $\mathrm{K}$ & $\mathrm{Ca}$ & $\mathrm{Mg}$ & $\mathrm{N}$ & $\mathrm{P}$ & $\bar{K}$ & $\mathrm{Ca}$ & $\mathrm{Mg}$ \\
\hline I & $0,22 \mathrm{~A}$ & $0,02 \mathrm{~A}$ & $0,04 \mathrm{~B}$ & $0,05 B$ & $0,02 \mathrm{AB}$ & $71,7 \mathrm{~B}$ & $1031,5 \mathrm{~A}$ & $1788 \mathrm{~A}$ & $293,14 \mathrm{~A}$ & $701,1 \mathrm{~A}$ \\
\hline II & $0,36 \mathrm{~A}$ & $0,03 \mathrm{~A}$ & $0,09 B$ & $0,08 B$ & $0,19 B$ & $76,6 \mathrm{AB}$ & $986,2 \mathrm{~A}$ & $328 \mathrm{~A}$ & $357,30 \mathrm{~A}$ & 1944,4 A \\
\hline III & $0,30 \mathrm{~A}$ & $0,03 \mathrm{~A}$ & $0,17 \mathrm{~A}$ & $0,14 \mathrm{~A}$ & $0,46 \mathrm{~A}$ & $92,2 \mathrm{~A}$ & $968,7 \mathrm{~A}$ & $159 \mathrm{~A}$ & $202,74 \mathrm{~A}$ & $615,7 \mathrm{~A}$ \\
\hline
\end{tabular}

Médias seguidas pela mesma letra não diferem significativamente entre si, pelo teste de Tukey (P>0,05).

Means followed by same letter do not differ significantly by Tukey test $(P>0.05)$.

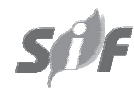

Revista Árvore, Viçosa-MG, v.34, n.6, p.991-999, 2010 
clima, variando com época do ano, idade e composição do material (SHANMUGHAVEL, 2004). O N, por ser o nutriente com maiores teores em folhas, foi o mais reciclado, concordando com Singh e Singh (1999) e Embaye et al. (2005).

A eficiência de utilização de nutrientes na composição da manta orgânica variou pouco entre povoamentos, sendo pouco afetada pela declividade e pela densidade de plantas (Tabela 7), variando apenas para $\mathrm{N}$, que é o nutriente menos eficientemente usado, pelo seu maior conteúdo nas folhas, o que pode facilitar a sua decomposição por microrganismos, já que estes se beneficiam de alta relação C : N (MELLO et al., 1987).

\section{5. Alocação de Nutrientes no Rizoma}

Com exceção do K, o conteúdo de nutrientes no rizoma não variou entre povoamentos, provavelmente porque, em todos os povoamentos, o rizoma se mantém estagnado, sob competição, já que é um componente perene da planta, por não ser explorado. Assim, a competição entre rizomas, que crescem continuamente, pode mudar resultados nutricionais na biomassa total, em comparação com a biomassa área exportada (Tabela 8).

O rizoma apresentou maiores conteúdos de nutrientes em relação aos componentes de parte aérea da planta. Apesar de ter o maior conteúdo individual de nutrientes, o conteúdo de nutrientes no rizoma não contém necessariamente mais nutrientes que a parte aérea total. Assim, foi validada a conclusão de Kleinhenz e Midmore (2001), ao rever a bibliografia mundial sobre bambu, de que o rizoma é componente formador de colmos e sistema de transporte que conecta colmos velhos e novos, pela drenagem de nutrientes recentemente absorvidos e metabolizados pela parte aérea. Assim, o rizoma não é órgão de armazenagem, e, devido ao rápido crescimento, não há armazenagem verdadeira de nutrientes no bambu. Assim, a manutenção de colmos novos, que mantêm e formam galhos e folhas, é importante para reduzir o tempo de interrupção do crescimento após o corte raso.

O N é o nutriente mais presente no rizoma, o que confirma a observação de Kleinhenz e Midmore (2001), que, ao revisarem a literatura mundial sobre bambu, concluíram que, abaixo do solo, nos rizomas, ocorre o maior conteúdo de $\mathrm{N}$.

A eficiência de utilização de $\mathrm{N}$ na produção de rizoma (Tabela 8) diferiu entre povoamentos, sendo os povoamentos I e III, sob maior densidade de plantas, mais eficientes, e para $\mathrm{K}$, em que o povoamento II, menos denso, foi mais eficiente. $\mathrm{O} \mathrm{N}$ foi também o nutriente menos eficientemente usado, o que é explicado pelo alto conteúdo nesse componente de biomassa.

\section{CONCLUSÕES}

O estudo da eficiência nutricional em plantios de bambu, que variavam na densidade de plantio e na declividade do terreno, permitiu as seguintes conclusões:

. A declividade elevou a absorção de P, K e Ca e a exportação de todos os nutrientes.

. A mais alta densidade de plantas elevou a absorção de $\mathrm{K}$, o elemento mais requerido.

. O K é o nutriente mais exportado pelo seu maior conteúdo e importância na nutrição da parte aérea, sendo, seguido do $\mathrm{N}$, Ca, P e Mg, especialmente importante no cálculo da adubação com base na sua eficiência de utilização pelas plantas.

. A eficiência de utilização de nutrientes na biomassa total e comercial não variou entre povoamentos, devido ao fato de a rotação curta determinar alta atividade metabólica, com elevado trânsito de nutrientes. A eficiência de absorção foi mais decisiva na produtividade.

Tabela 8 - Conteúdo médio e CUB de nutrientes no rizoma de povoamentos de Bambusa vulgaris, em Pedras de Fogo, PB. Table 8 - Medium Content and CUB of Nutrients in the Rhizome, in Commercial Stands of Bambusa vulgaris, in Pedras de Fogo, $P B$.

\begin{tabular}{|c|c|c|c|c|c|c|c|c|c|c|}
\hline \multirow[t]{2}{*}{ Povoamentos } & \multicolumn{5}{|c|}{ Conteúdo de nutrientes (kg/ha) } & \multicolumn{5}{|c|}{ CUB (kg matéria seca/kg nutriente) } \\
\hline & $\mathrm{N}$ & $\mathrm{P}$ & $\mathrm{K}$ & $\mathrm{Ca}$ & $\mathrm{Mg}$ & $\mathrm{N}$ & $\mathrm{P}$ & $\mathrm{K}$ & $\mathrm{Ca}$ & $\mathrm{Mg}$ \\
\hline $\mathrm{I}$ & $118,6 \mathrm{~A}$ & $10,8 \mathrm{~A}$ & $82,3 \mathrm{~A}$ & $32,8 \mathrm{~A}$ & $19,0 \mathrm{~A}$ & $148,7 \mathrm{~A}$ & 3393A & $197,8 \mathrm{~B}$ & $831,7 \mathrm{~A}$ & $1838,6 \mathrm{~A}$ \\
\hline II & $91,6 \mathrm{~A}$ & 7,7A & $7,8 \mathrm{~B}$ & $23,8 \mathrm{~A}$ & $8,6 \mathrm{~A}$ & $106,5 B$ & 1235A & $1514,3 \mathrm{~A}$ & $420,1 \mathrm{~A}$ & $1350,7 \mathrm{~A}$ \\
\hline III & $67,1 \mathrm{~A}$ & $9,4 \mathrm{~A}$ & $60,4 \mathrm{~A}$ & $34,4 \mathrm{~A}$ & $9,0 \mathrm{~A}$ & $151,7 \mathrm{~A}$ & 1058A & $186,6 \mathrm{~B}$ & $698,7 \mathrm{~A}$ & $1306,7 \mathrm{~A}$ \\
\hline C. V. (\%) & 65,6 & 60,1 & 53,1 & 64,9 & 78,9 & 23,2 & 181,3 & 113,8 & 145,4 & 121,8 \\
\hline
\end{tabular}

Médias seguidas pela mesma letra não diferem significativamente entre si, pelo teste de Tukey (P>0,05).

Means followed by same letter do not differ significantly by Tukey test $(P>0.05)$.

Revista Árvore, Viçosa-MG, v.34, n.6, p.991-999, 2010 
. A quantidade de nutrientes devolvida ao solo pelas folhas em rotação curta não variou entre povoamentos, mas é importante em especial para N. A eficiência de utilização de nutrientes nas folhas variou apenas para o P. Como a reposição de nutrientes não compensa a exportação de nutrientes, a fertilização é importante para a manutenção da produtividade.

. A quantidade de nutrientes na manta orgânica pode variar entre povoamentos, sendo maior no povoamento em declive e para N. A eficiência de utilização de nutrientes na manta orgânica variou apenas para N, elemento-chave na decomposição orgânica.

. O rizoma exibiu os maiores conteúdos de nutrientes, em relação a cada componente de biomassa; $\mathrm{N}$ foi menos eficientemente usado no rizoma, por seu relativamente alto conteúdo.

\section{REFERÊNCIAS}

ARUNACHALAM, A.; ARUNACHALAM, K. Evaluation of bamboos in eco-restoration of "jhum" fallows in Arunachal Pradesh: ground vegetation, soil and microbial biomass. Forest Ecology and Management, v.159, n.3, p.231-239, 2002.

BARROS, N. F. et al. Produção de eucalipto em solos de cerrados em resposta à aplicação de NPK e de B e Zn. Revista Árvore, v.5, n.1, p.90-103, 1981.

BARROS, N. F. et al. Interpretação de análises químicas de solo para o crescimento de Eucalyptus spp. Revista Árvore, v.6, n.1, p.38-44, 1982.

BARROS, N. F. et al. Classificação nutricional de sítios florestais - descrição de uma metodologia. Revista Árvore, v.10, n.1, p.112-20, 1986.

BARROS, N. F.; NOVAIS, R. F.; NEVES, J. C. L. Fertilização e correção do solo para o plantio de eucalipto. In: BARROS, N. F.; NOVAIS, R. F. (Eds.). Relação solo-eucalipto. Viçosa, MG: Folha de Viçosa, 1990. p.1-24.

BRAGA, J. M.; DEFELIPO, B. V. Determinação espectrofotométrica de fósforo em extratos de solos e plantas. Revista Ceres, v.21, n.113, p.73-85, 1974.

CHRISTANTY, L.; MAILLY, D.; KIMMINS, J. P. "Without bamboo, the land dies": Biomass, litterfall, and soil organic matter dynamics of a Javanese bamboo talon-kebun system. Forest Ecology and Management, v.87, n.1, p.75-88, 1996.
EMBAYE, K. et al. Biomass and nutrient distribution in a highland bamboo forest in southwest Ethiopia: implications for management. Forest Ecology and Management, v.204, n.2/3, p.159-169, 2005.

FRANÇA, F. S.; POGGIANI, F. Distribuição de biomassa e dos macronutrientes em três espécies de eucalipto plantadas em diferentes espaçamentos. In: BARROS, N. F. (Coord.). FLORESTAS PLANTADAS NOS NEOTRÓPICOS COMO FONTE DE ENERGIA, 1983, VIÇOSA. Resumos... Viçosa,MG: Universidade Federal de Viçosa, 1983. p.450-451.

KLEINHENZ, V.; MIDMORE, D. J. Aspects of bamboo agronomy. Advances in Agronomy, v.74, p.99-149, 2001.

MELLO, F. De A. F. de et alli. Fertilidade do solo. Piracicaba, Nobel, 1987. 400p.

MOURA, O. N. Distribuição de Biomassa, nutrientes e eficiência nutricional em povoamentos de sabiá (Mimosa caesalpinifolia Benth.). 1999. 52f. Dissertação (Mestrado em Solos) - Universidade Federal Rural de Pernambuco, Recife, 1999.

REZENDE, G. C. et al. Produção e macronutrientes em florestas de eucalipto sob duas densidades. Revista Árvore, v.7, n.2, p.165-176, 1983.

SINGH,A. N.; SINGH, J. S. Biomass, net primary production and impact of bamboo plantation on soil redevelopment in a dry tropical region. Forest Ecology and Management, v.119, p.195-207, 1999.

SHANMUGHAVEL, P.; FRANCIS, K. Biomass and nutrient cycling in bamboo (Bambusa bambos) plantations of tropical areas. Biology and Fertility of Soils, v.23, n.4, p.431-434, 1996.

SHANMUGHAVEL, P.; FRANCIS, K. The dynamics of biomass and nutrientes in bamboo (Bambusa vulgaris) plantations. Journal of Bamboo and Rattan, v.1, n.2. p.157-170, 2002.

SHANMUGHAVEL, P. Litter decomposition and nutrient release in a bamboo plantation.

Germantown, NY: Journal of Bamboo and Rattan, v.3, n.4, p.319-328, 2004.

TEIXEIRA, J. L. Conteúdo de nutrientes e produção de eucalipto em diferentes ambientes do Rio Doce - MG. 1987. $70 \mathrm{f}$. Dissertação (Mestrado em Solos) - Universidade Federal de Viçosa, Viçosa, MG, 1987.

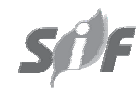

Revista Árvore, Viçosa-MG, v.34, n.6, p.991-999, 2010 